\title{
Gröbli solution for three magnetic vortices
}

\author{
Stavros Komineas ${ }^{1}$ and Nikos Papanicolaou ${ }^{2,3}$ \\ ${ }^{1}$ Department of Applied Mathematics, University of Crete, 71409 Heraklion, Crete, Greece \\ ${ }^{2}$ Department of Physics, University of Crete, 71003 Heraklion, Crete, Greece \\ ${ }^{3}$ Institute for Theoretical and Computational Physics, University of Crete, Heraklion, Greece
}

The dynamics of $N$ point vortices in a fluid is described by the Helmholtz-Kirchhoff (HK) equations which lead to a completely integrable Hamiltonian system for $N=2$ or 3 but chaotic dynamics for $N>3$. Here we consider a generalization of the HK equations to describe the dynamics of magnetic vortices within a collective-coordinate approximation. In particular, we analyze in detail the dynamics of a system of three magnetic vortices by a suitable generalization of the solution for three point vortices in an ordinary fluid obtained by Gröbli more than a century ago. The significance of our results for the dynamics of ferromagnetic elements is briefly discussed.

\section{INTRODUCTION}

Ferromagnetic media are described by the density of magnetic moment or magnetization $\boldsymbol{m}=\boldsymbol{m}(\boldsymbol{r}, t)$ which is a vector field of constant length that satisfies the highly nonlinear LandauLifshitz (LL) equation. Magnetic bubbles and vortices are special solutions of the LL equation characterized by a topological invariant sometimes called the Pontryagin index or skyrmion number. Upon suitable normalization, the skyrmion number is integer for magnetic bubbles occuring in magnetic films with perpendicular easy-axis anisotropy [1] and half integer for magnetic vortices that may be realized in the case of magnetic films with easy-plane anistotropy [2].

Most of the early studies were carried out on magnetic films of infinite extent where vortices and bubbles occur as excited states above a uniform ground state. More recently it has been realized that the ground state of a disc-shaped magnetic element, with a diameter of a few hundred nanometers, is itself a vortex configuration. Hence the vortex is a nontrivial magnetic state that can be spontaneously created on magnetic elements of finite extent. Such a possibility is of obvious significance for device applications and explains the current interest on this subject 3, 4, [5].

The dynamics of magnetic vortices is greatly affected by the underlying topological structure $\underline{6}$, 7]. This is described by a topological vorticity which gives the skyrmion number upon integration over the plane of the magnetic film. As a result, the essential features of magnetic vortex dynamics are similar to those displayed by ordinary vortices in fluid dynamics. A single vortex or antivortex is spontaneously pinned and thus cannot move freely within the ferromagnetic medium. However, vortex motion relative to the medium is possible in the presence of other vortices, or externally applied magnetic field gradients, and displays characteristics analogous to those of two-dimensional (2D) motion of electric charges in a uniform magnetic field. In particular, two like vortices orbit around each other while a vortex-antivortex pair undergoes Kelvin motion. A recent review of two-vortex magnetic systems together with some progress in the study of three-vortex dynamics may be found in Refs. [8, 9].

In the present paper we study the dynamics of a system consisting of $N$ interacting magnetic vortices within a collective-coordinate approximation which evades the complexities of the complete LL equation but retains important dynamical features due to the underlying topology. The relevant equations are a generalization of the HK equations encountered in the study of $N$ point vortices in ordinary fluids [10, 11]. The latter equations were solved explicitly by Helmholtz himself for the two-vortex $(N=2)$ system, while Kirchhoff concluded that the three-vortex $(N=3)$ system is completely integrable. But a complete solution for $N=3$ was given in the 1877 dissertation of Gröbli [12] recently reviewed by Aref et al [13] (see also [14]). For $N>3$, the system is not completely integrable and thus leads to chaotic dynamics. 
In Section II, we state the appropriate modifications of the HK equations to account for $N$ interacting magnetic vortices, derive the corresponding conservation laws, and provide a first demonstration with an explicit solution for two-vortex systems. The generalization of Gröbli's solution to three magnetic vortices is given in Section III and is illustrated in detail in Section IV for important special cases such as scattering of a vortex-antivortex pair off a target vortex initially at rest, three-vortex collapse, bounded three-vortex motion, etc. Our main conclusions are summarized in Section V.

\section{GENERALIZATION OF THE HELMHOLTZ-KIRCHHOFF EQUATIONS}

We consider a system of $N$ magnetic vortices labeled by $\alpha=1,2, \ldots N$. Each vortex is characterized by a pair of indices $\left(\kappa_{\alpha}, \lambda_{\alpha}\right)$ where $\kappa_{\alpha}$ is the vortex number and $\lambda_{\alpha}$ the polarity, which take the values $\kappa_{\alpha}= \pm 1$ and $\lambda_{\alpha}= \pm 1$ in any combination. We also define the skyrmion number

$$
s_{\alpha}=\kappa_{\alpha} \lambda_{\alpha}
$$

which also takes the distinct values $s_{\alpha}= \pm 1$ (in any combination for varying $\alpha=1,2, \ldots N$ ) and differs from the standard definition of the Pontryagin index by an overall normalization $\left(N_{\alpha}=-\frac{1}{2} s_{\alpha}\right)[\underline{8}, 9]$.

Now, in a collective-coordinate approximation [15, 16, 17], the position of each vortex is labeled by a vector $\boldsymbol{r}_{\alpha}=\left(x_{\alpha}, y_{\alpha}\right)$ in the $2 \mathrm{D}$ plane and the energy functional is given by

$$
E=-\sum_{\alpha<\beta} \kappa_{\alpha} \kappa_{\beta} \ln \left|\boldsymbol{r}_{\alpha}-\boldsymbol{r}_{\beta}\right|
$$

in suitable units. Note that the energy is a function of the vortex number $\kappa_{\alpha}$ but not the polarities $\lambda_{\alpha}$. The equations of motion are then written in Hamiltonian form as

$$
s_{\alpha} \frac{d x_{\alpha}}{d t}=\frac{\partial E}{\partial y_{\alpha}}, \quad s_{\alpha} \frac{d y_{\alpha}}{d t}=-\frac{\partial E}{\partial x_{\alpha}} ; \quad \alpha=1,2, \ldots N,
$$

or, more explicitly, as

$$
\lambda_{\alpha} \frac{d x_{\alpha}}{d t}=-\sum_{\beta \neq \alpha} \kappa_{\beta} \frac{y_{\alpha}-y_{\beta}}{\left|\boldsymbol{r}_{\alpha}-\boldsymbol{r}_{\beta}\right|^{2}}, \quad \lambda_{\alpha} \frac{d y_{\alpha}}{d t}=\sum_{\beta \neq \alpha} \kappa_{\beta} \frac{x_{\alpha}-x_{\beta}}{\left|\boldsymbol{r}_{\alpha}-\boldsymbol{r}_{\beta}\right|^{2}} .
$$

These differ from the HK equations for $N$ point vortices in an ordinary fluid by the presence of the polarities $\lambda_{\alpha}$ (or the skyrmion numbers $s_{\alpha}$ ) in addition to the vortex numbers $\kappa_{\alpha}$. Equations (4) reduce to the HK equations in the special limit where all polarities are taken to be equal $\left(\lambda_{1}=\lambda_{2}=\ldots=\lambda_{N}\right)$. Also note that the vortex number $\kappa_{\alpha}$ may assume any continuous value for a fluid vortex but takes the quantized values \pm 1 for a magnetic vortex.

In addition to the conserved energy of Eq. (2), the Hamiltonian system (3) or (44) possesses conservation laws which originate in translational and rotational invariance; i.e., the analog of linear momentum $\boldsymbol{P}=\left(P_{x}, P_{y}\right)$ with

$$
P_{x}=-\sum_{\alpha} s_{\alpha} y_{\alpha}, \quad P_{y}=\sum_{\alpha} s_{\alpha} x_{\alpha},
$$

and angular momentum

$$
L=\frac{1}{2} \sum_{\alpha} s_{\alpha}\left(x_{\alpha}^{2}+y_{\alpha}^{2}\right)
$$


both defined in convenient units. Actually, the physical significance of these conservation laws depends crucially on whether or not the total skyrmion number

$$
S=\sum_{\alpha} s_{\alpha}=\sum_{\alpha} \kappa_{\alpha} \lambda_{\alpha}
$$

vanishes. The linear momentum (5) assumes its customary meaning only when $S=0$. For $S \neq 0$, one may instead define the conserved guiding center $\boldsymbol{R}=\left(R_{x}, R_{y}\right)$ with

$$
R_{x}=\frac{1}{S} \sum_{\alpha} s_{\alpha} x_{\alpha}, \quad R_{y}=\frac{1}{S} \sum_{\alpha} s_{\alpha} y_{\alpha},
$$

which is a measure of position rather than linear momentum. The inherent transmutation of momentum into position occurs also within the complete LL equation and has been the subject of several investigations [6, 7, 9]. To complete the discussion of conservation laws we note that the linear momentum (5) and the angular momentum (6) may be combined to yield the derivative conserved quantity

$$
\frac{1}{2} \sum_{\alpha \beta} s_{\alpha} s_{\beta}\left(\boldsymbol{r}_{\alpha}-\boldsymbol{r}_{\beta}\right)^{2}
$$

which depends only on the relative distances $\left|\boldsymbol{r}_{\alpha}-\boldsymbol{r}_{\beta}\right|$ and will play an important role in the following.

This section is concluded with a simple application of the general formalism to the case of two-vortex systems $(N=2)$. Equations (4) then read

$$
\begin{aligned}
\lambda_{1} \frac{d x_{1}}{d t} & =-\kappa_{2} \frac{y_{1}-y_{2}}{d^{2}}, & \lambda_{2} \frac{d x_{2}}{d t} & =-\kappa_{1} \frac{y_{2}-y_{1}}{d^{2}}, \\
\lambda_{1} \frac{d y_{1}}{d t} & =\kappa_{2} \frac{x_{1}-x_{2}}{d^{2}}, & \lambda_{2} \frac{d y_{2}}{d t} & =\kappa_{1} \frac{x_{2}-x_{1}}{d^{2}},
\end{aligned}
$$

where $d^{2} \equiv\left(x_{2}-x_{1}\right)^{2}+\left(y_{2}-y_{1}\right)^{2}$ is the squared relative distance between the two vortices which is conserved by virtue of the conservation of the energy $E$ of Eq. (2) restricted to $N=2$ for any choice of vortex numbers and polarities. But the details of the motion depend on the specific choice of $\kappa$ 's and $\lambda$ 's as demonstrated below with two representative examples.

First, we consider a vortex-antivortex pair with equal polarities:

$$
\left(\kappa_{1}, \lambda_{1}\right)=(1,1), \quad\left(\kappa_{2}, \lambda_{2}\right)=(-1,1),
$$

hence opposite skyrmion numbers $s_{1}=1, s_{2}=-1$ and total skyrmion number $S=s_{1}+s_{2}=0$. A straightforward solution of Eqs. (10) then leads to Kelvin motion where the vortex and the antivortex move in formation at constant relative distance $d$, along parallel trajectories that are perpendicular to the line connecting the vortex and the antivortex, with speed

$$
V=\frac{1}{d}
$$

and linear momentum (5) that is parallel to the velocity.

In contrast, a vortex-antivortex pair with opposite polarities:

$$
\left(\kappa_{1}, \lambda_{1}\right)=(-1,1), \quad\left(\kappa_{2}, \lambda_{2}\right)=(1,-1),
$$

hence $s_{1}=s_{2}=-1$ and nonvanishing total skyrmion number $S=s_{1}+s_{2}=-2$, undergoes rotational motion at constant diameter $d$, around a fixed guiding center (8) which may be taken to be the origin of the coordinate system $\left(x_{1}+x_{2}=0=y_{1}+y_{2}\right)$, with angular frequency

$$
\omega=\frac{2}{d^{2}} \text {. }
$$


A detailed study of Kelvin and rotational motion was carried out within the complete LL equation in Refs. [18] and [19], respectively, the results of which were recently reviewed in Ref. [8]. In particular, their results confirm the validity of Eqs. (12) and (14) for sufficiently large relative distance $d$, as expected for the collective-coordinate approximation adopted in the present paper.

Finally we note the the motion of a two-vortex system is Kelvin-like when the total skyrmion number vanishes $\left(S=s_{1}+s_{2}=\kappa_{1} \lambda_{1}+\kappa_{2} \lambda_{2}=0\right)$ and rotational otherwise $\left(S=s_{1}+s_{2}=\right.$ $\kappa_{1} \lambda_{1}+\kappa_{2} \lambda_{2}= \pm 2$ ) irrespectively of the detailed configuration of the vortex numbers $\kappa_{1}, \kappa_{2}$ and polarities $\lambda_{1}, \lambda_{2}$.

\section{THREE MAGNETIC VORTICES}

Consider a system of three interacting magnetic vortices with vortex numbers and polarities given by the three pairs of indices $\left(\kappa_{1}, \lambda_{1}\right),\left(\kappa_{2}, \lambda_{2}\right),\left(\kappa_{3}, \lambda_{3}\right)$. The three vortices form a triangle whose vertices are located at $\boldsymbol{r}_{1}=\left(x_{1}, y_{1}\right), \boldsymbol{r}_{2}=\left(x_{2}, y_{2}\right), \boldsymbol{r}_{3}=\left(x_{3}, y_{3}\right)$ and move about the 2D plane according to Eqs. (4) written here explicitly as

$$
\begin{array}{llrl}
\lambda_{1} \frac{d x_{1}}{d t} & =-\kappa_{2} \frac{y_{1}-y_{2}}{C_{3}^{2}}+\kappa_{3} \frac{y_{3}-y_{1}}{C_{2}^{2}}, & \lambda_{1} \frac{d y_{1}}{d t}=\kappa_{2} \frac{x_{1}-x_{2}}{C_{3}^{2}}-\kappa_{3} \frac{x_{3}-x_{1}}{C_{2}^{2}} \\
\lambda_{2} \frac{d x_{2}}{d t}=-\kappa_{3} \frac{y_{2}-y_{3}}{C_{1}^{2}}+\kappa_{1} \frac{y_{1}-y_{2}}{C_{3}^{2}}, & \lambda_{2} \frac{d y_{2}}{d t}=\kappa_{3} \frac{x_{2}-x_{3}}{C_{1}^{2}}-\kappa_{1} \frac{x_{1}-x_{2}}{C_{3}^{2}} \\
\lambda_{3} \frac{d x_{3}}{d t}=-\kappa_{1} \frac{y_{3}-y_{1}}{C_{2}^{2}}+\kappa_{2} \frac{y_{2}-y_{3}}{C_{1}^{2}}, & \lambda_{3} \frac{d y_{3}}{d t}=\kappa_{1} \frac{x_{3}-x_{1}}{C_{2}^{2}}-\kappa_{2} \frac{x_{2}-x_{3}}{C_{1}^{2}}
\end{array}
$$

where

$$
C_{1}=\left|\boldsymbol{r}_{2}-\boldsymbol{r}_{3}\right|, \quad C_{2}=\left|\boldsymbol{r}_{3}-\boldsymbol{r}_{1}\right|, \quad C_{3}=\left|\boldsymbol{r}_{1}-\boldsymbol{r}_{2}\right|
$$

are the lengths of the triangle sides.

Our task is to solve the initial-value problem defined by Eqs. (15) with initial conditions furnished by the $t=0$ configuration of the three vortices. Needless to say, a numerical solution is straightforward and has indeed been used to confirm our main analytical results obtained in the following by a generalization of Gröbli's original solution for ordinary fluid vortices [12].

The key observation is that the scalar quantities $C_{1}, C_{2}$ and $C_{3}$ satisfy a closed system of equations of their own, which may be employed to determine the time evolution of the shape and size of the vortex triangle, irrespectively of its relative orientation and motion in the plane. Specifically, as a consequence of Eqs. (15),

$$
\begin{aligned}
& \frac{d}{d t}\left(C_{1}^{2}\right)=4 \kappa_{1} A\left(\frac{1}{\lambda_{3} C_{2}^{2}}-\frac{1}{\lambda_{2} C_{3}^{2}}\right) \\
& \frac{d}{d t}\left(C_{2}^{2}\right)=4 \kappa_{2} A\left(\frac{1}{\lambda_{1} C_{3}^{2}}-\frac{1}{\lambda_{3} C_{1}^{2}}\right) \\
& \frac{d}{d t}\left(C_{3}^{2}\right)=4 \kappa_{3} A\left(\frac{1}{\lambda_{2} C_{1}^{2}}-\frac{1}{\lambda_{1} C_{2}^{2}}\right) .
\end{aligned}
$$

Here $A=\nu|A|$ where $\nu=1$ or -1 when the vortices $(1,2,3)$ appear in the counterclockwise or clockwise sense in the plane, and $|A|$ is the area of the vortex triangle which may be expressed entirely in terms of $C_{1}, C_{2}$ and $C_{3}$ :

$$
|A|=\left[C\left(C-C_{1}\right)\left(C-C_{2}\right)\left(C-C_{3}\right)\right]^{1 / 2}, \quad C \equiv \frac{1}{2}\left(C_{1}+C_{2}+C_{3}\right) .
$$


Eqs. (17) constistute a reduced dynamical system that is completely integrable because it possesses two conservation laws; namely

$$
B=C_{1}^{1 / \kappa_{1}} C_{2}^{1 / \kappa_{2}} C_{3}^{1 / \kappa_{3}}, \quad \Gamma=\frac{s_{2} s_{3} C_{1}^{2}+s_{3} s_{1} C_{2}^{2}+s_{1} s_{2} C_{3}^{2}}{s_{1}+s_{2}+s_{3}},
$$

where the conservation of $B$ follows from the conservation of the energy of Eq. (21), and $\Gamma$ from the conservation of the quantity defined in Eq. (9), both restricted to the three-vortex system and normalized in a manner suitable for subsequent analysis. The conservation of $B$ and $\Gamma$ may be confirmed directly from Eqs. (17). Thus, having determined $B$ and $\Gamma$ from the initial $(t=0)$ values of $C_{1}, C_{2}$ and $C_{3}$, one may use Eqs. (19) to eliminate two of these variables, say, $C_{2}$ and $C_{3}$ in favor of $C_{1}$ and thereby reduce Eqs. (17) to a single equation for $C_{1}$ that may be integrated by a simple quadrature.

In order to complete the solution we must also determine the relative orientation and motion of the vortex triangle in the plane and thus solve for the actual vortex trajectories. The latter may be conveniently described in polar coordinates $\left(r_{\alpha}, \theta_{\alpha}\right), \alpha=1,2,3$. Using these coordinates Hamilton's equations (3) read

$$
s_{\alpha} r_{\alpha} \frac{d r_{\alpha}}{d t}=\frac{\partial E}{\partial \theta_{\alpha}} \quad s_{\alpha} r_{\alpha} \frac{d \theta_{\alpha}}{d t}=-\frac{\partial E}{\partial r_{\alpha}} .
$$

We write explicitly the three equations for the polar angles:

$$
\begin{aligned}
& \lambda_{1} r_{1} \frac{d \theta_{1}}{d t}=\kappa_{2} \frac{r_{1}-r_{2} \cos \left(\theta_{1}-\theta_{2}\right)}{C_{3}^{2}}+\kappa_{3} \frac{r_{1}-r_{3} \cos \left(\theta_{3}-\theta_{1}\right)}{C_{2}^{2}}, \\
& \lambda_{2} r_{2} \frac{d \theta_{2}}{d t}=\kappa_{3} \frac{r_{2}-r_{3} \cos \left(\theta_{2}-\theta_{3}\right)}{C_{1}^{2}}+\kappa_{1} \frac{r_{2}-r_{1} \cos \left(\theta_{1}-\theta_{2}\right)}{C_{3}^{2}}, \\
& \lambda_{3} r_{3} \frac{d \theta_{3}}{d t}=\kappa_{1} \frac{r_{3}-r_{1} \cos \left(\theta_{3}-\theta_{1}\right)}{C_{2}^{2}}+\kappa_{2} \frac{r_{3}-r_{2} \cos \left(\theta_{2}-\theta_{3}\right)}{C_{1}^{2}} .
\end{aligned}
$$

A crucial step for simplifying the latter equations is to set the guiding center (8) at the origin, i.e., $R_{x}=0=R_{y}$. In polar coordinates this constraint reads:

$$
\begin{aligned}
s_{1} r_{1} \cos \theta_{1}+s_{2} r_{2} \cos \theta_{2}+s_{3} r_{3} \cos \theta_{3} & =0 \\
s_{1} r_{1} \sin \theta_{1}+s_{2} r_{2} \sin \theta_{2}+s_{3} r_{3} \sin \theta_{3} & =0 .
\end{aligned}
$$

Simple combinations of Eqs. (22) yield

$$
\begin{aligned}
& 2 s_{2} s_{3} r_{2} r_{3} \cos \left(\theta_{2}-\theta_{3}\right)=s_{1}^{2} r_{1}^{2}-s_{2}^{2} r_{2}^{2}-s_{3}^{2} r_{3}^{2} \\
& 2 s_{3} s_{1} r_{3} r_{1} \cos \left(\theta_{3}-\theta_{1}\right)=-s_{1}^{2} r_{1}^{2}+s_{2}^{2} r_{2}^{2}-s_{3}^{2} r_{3}^{2} \\
& 2 s_{1} s_{2} r_{1} r_{2} \cos \left(\theta_{1}-\theta_{2}\right)=-s_{1}^{2} r_{1}^{2}-s_{2}^{2} r_{2}^{2}+s_{3}^{2} r_{3}^{2} .
\end{aligned}
$$

Further, we write the sides of the triangle using polar coordinates:

$$
\begin{aligned}
& C_{1}^{2}=r_{2}^{2}+r_{3}^{2}-2 r_{2} r_{3} \cos \left(\theta_{2}-\theta_{3}\right) \\
& C_{2}^{2}=r_{3}^{2}+r_{1}^{2}-2 r_{3} r_{1} \cos \left(\theta_{3}-\theta_{1}\right) \\
& C_{3}^{2}=r_{1}^{2}+r_{2}^{2}-2 r_{1} r_{2} \cos \left(\theta_{1}-\theta_{2}\right),
\end{aligned}
$$

and we can now use Eqs. (23) and (24) to obtain explicit relations between the radial coordinates $r_{\alpha}$ and the sides of the triangle $C_{\alpha}$ :

$$
\begin{aligned}
& s_{2} s_{3} C_{1}^{2}=\left(s_{2}+s_{3}\right) \Gamma-s_{1}\left(s_{1}+s_{2}+s_{3}\right) r_{1}^{2} \\
& s_{3} s_{1} C_{2}^{2}=\left(s_{3}+s_{1}\right) \Gamma-s_{2}\left(s_{1}+s_{2}+s_{3}\right) r_{2}^{2} \\
& s_{1} s_{2} C_{3}^{2}=\left(s_{1}+s_{2}\right) \Gamma-s_{3}\left(s_{1}+s_{2}+s_{3}\right) r_{3}^{2},
\end{aligned}
$$


where $\Gamma$ is the conserved quantity already defined in Eq. (19).

We are now ready to write Eqs. (21) in a form where the radial coordinates $r_{\alpha}$ are eliminated and only the variables $C_{\alpha}$ and $\theta_{\alpha}$ appear. We use Eqs. (24) to eliminate the cosines and Eqs. (25) to express the radial coordinates in terms of the $C_{\alpha}$ 's in Eqs. (21), to obtain

$$
\begin{aligned}
& 2 \lambda_{1} \lambda_{2} \lambda_{3}\left(s_{1}+s_{2}+s_{3}\right) r_{1}^{2} C_{2}^{2} C_{3}^{2} \frac{d \theta_{1}}{d t}=\lambda_{3} C_{2}^{2}\left[s_{2} s_{3}\left(-C_{1}^{2}+C_{2}^{2}+C_{3}^{2}\right)+2 s_{2}^{2} C_{3}^{2}\right] \\
&+\lambda_{2} C_{3}^{2}\left[s_{2} s_{3}\left(-C_{1}^{2}+C_{2}^{2}+C_{3}^{2}\right)+2 s_{3}^{2} C_{2}^{2}\right] \\
& 2 \lambda_{1} \lambda_{2} \lambda_{3}\left(s_{1}+s_{2}+s_{3}\right) r_{2}^{2} C_{3}^{2} C_{1}^{2} \frac{d \theta_{2}}{d t}=\lambda_{1} C_{3}^{2}\left[s_{3} s_{1}\left(C_{1}^{2}-C_{2}^{2}+C_{3}^{2}\right)+2 s_{3}^{2} C_{1}^{2}\right] \\
&+\lambda_{3} C_{1}^{2}\left[s_{3} s_{1}\left(C_{1}^{2}-C_{2}^{2}+C_{3}^{2}\right)+2 s_{1}^{2} C_{3}^{2}\right] \\
& 2 \lambda_{1} \lambda_{2} \lambda_{3}\left(s_{1}+s_{2}+s_{3}\right) r_{3}^{2} C_{1}^{2} C_{2}^{2} \frac{d \theta_{3}}{d t}= \lambda_{2} C_{1}^{2}\left[s_{1} s_{2}\left(C_{1}^{2}+C_{2}^{2}-C_{3}^{2}\right)+2 s_{1}^{2} C_{2}^{2}\right] \\
&+\lambda_{1} C_{2}^{2}\left[s_{1} s_{2}\left(C_{1}^{2}+C_{2}^{2}-C_{3}^{2}\right)+2 s_{2}^{2} C_{1}^{2}\right]
\end{aligned}
$$

where it is understood that the $r_{\alpha}$ 's are given through Eqs. 25) in terms of the $C_{\alpha}$ 's. Explicit applications of the preceding general results are given in Section IV.

\section{AN INTERESTING THREE-VORTEX SYSTEM}

For an explicit demonstration we consider a three-vortex system with vortex numbers and polarities given by

$$
\left(\kappa_{1}, \lambda_{1}\right)=(1,1), \quad\left(\kappa_{2}, \lambda_{2}\right)=(-1,1), \quad\left(\kappa_{3}, \lambda_{3}\right)=(1,-1)
$$

and corresponding skyrmion numbers $s_{1}=1, s_{2}=-1=s_{3}$. The pair $(1,2)$ is the vortex -antivortex pair of Eq. (11) which would undergo Kelvin motion in the absence of vortex 3 . In contrast, the pair $(2,3)$ would undergo rotational motion in the absence of vortex 1 , as discussed in Sec. II following Eq. (13). Thus the motion of the three vortices is expected to be a combination of Kelvin and rotational motion. Since the total skyrmion number $S=s_{1}+s_{2}+s_{3}=-1$ is different from zero, the guiding center for the three-vortex system defined from Eq. (8) , i.e.,

$$
R_{x}=-x_{1}+x_{2}+x_{3}, \quad R_{y}=-y_{1}+y_{2}+y_{3},
$$

is conserved and thus remains fixed during the motion.

We now return to the reduced system (17) applied for the specific choice (27):

$$
\begin{aligned}
& \frac{d}{d t}\left(C_{1}^{2}\right)=-4 A\left(\frac{1}{C_{2}^{2}}+\frac{1}{C_{3}^{2}}\right) \\
& \frac{d}{d t}\left(C_{2}^{2}\right)=-4 A\left(\frac{1}{C_{3}^{2}}+\frac{1}{C_{1}^{2}}\right) \\
& \frac{d}{d t}\left(C_{3}^{2}\right)=4 A\left(\frac{1}{C_{1}^{2}}-\frac{1}{C_{2}^{2}}\right)
\end{aligned}
$$

while the conservation laws defined from Eq. (19) are given by

$$
B=\frac{C_{1} C_{3}}{C_{2}}, \quad \Gamma=-C_{1}^{2}+C_{2}^{2}+C_{3}^{2} .
$$


One may then express $C_{2}$ and $C_{3}$ in terms of $C_{1}$ as

$$
C_{2}^{2}=\frac{C_{1}^{2}+\Gamma}{C_{1}^{2}+B^{2}} C_{1}^{2}, \quad C_{3}^{2}=\frac{C_{1}^{2}+\Gamma}{C_{1}^{2}+B^{2}} B^{2}
$$

and the area of the triangle defined earlier in Eq. (18) as

$$
4 A=\nu \sqrt{4 C_{2}^{2} C_{3}^{2}-\Gamma^{2}}=\frac{\nu}{C_{1}^{2}+B^{2}} \sqrt{4 B^{2} C_{1}^{2}\left(C_{1}^{2}+\Gamma\right)^{2}-\Gamma^{2}\left(C_{1}^{2}+B^{2}\right)^{2}} .
$$

Then the system of Eqs. (29) reduces to the single equation

$$
\frac{d}{d t}\left(C_{1}^{2}\right)=-\frac{\nu}{C_{1}^{2}+\Gamma}\left(\frac{1}{C_{1}^{2}}+\frac{1}{B^{2}}\right) \sqrt{4 B^{2} C_{1}^{2}\left(C_{1}^{2}+\Gamma\right)^{2}-\Gamma^{2}\left(C_{1}^{2}+B^{2}\right)^{2}},
$$

which may readily be integrated to yield $C_{1}=C_{1}(t)$, as well as $C_{2}=C_{2}(t)$ and $C_{3}=C_{3}(t)$ from Eq. (31), for any specific values of the conserved quantities $B$ and $\Gamma$ calculated from the initial three-vortex configuration.

In order to complete the solution we must also determine the relative orientation and motion of the vortex triangle in the plane and thus solve for the actual vortex trajectories. We will follow the derivation given in Section III and quote only important results that will allow us to provide a detailed illustration of the three-vortex motion for the special case of vortex numbers and polarities given by Eq. (27).

It is convenient to specify a reference frame such that its origin coincides with the conserved guiding center of Eq. (28):

$$
-x_{1}+x_{2}+x_{3}=0, \quad-y_{1}+y_{2}+y_{3}=0 .
$$

In this frame the radial distances $r_{1}, r_{2}$ and $r_{3}$ are related to the triangle sides $C_{1}, C_{2}$ and $C_{3}$ by Eq. (25) applied for $s_{1}=1, s_{2}=-1=s_{3}$ :

$$
r_{1}^{2}=2\left(C_{2}^{2}+C_{3}^{2}\right)-C_{1}^{2}, \quad r_{2}^{2}=C_{2}^{2}, \quad r_{3}^{2}=C_{3}^{2},
$$

while the angular variables satisfy Eqs. (26) now reduced to

$$
\begin{aligned}
\frac{d \theta_{1}}{d t} & =\frac{\Gamma}{2\left(C_{1}^{2}+2 \Gamma\right)}\left(\frac{1}{C_{2}^{2}}-\frac{1}{C_{3}^{2}}\right) \\
\frac{d \theta_{2}}{d t} & =-\frac{\Gamma}{2 C_{2}^{2}}\left(\frac{1}{C_{1}^{2}}+\frac{1}{C_{3}^{2}}\right)+\frac{1}{C_{1}^{2}} \\
\frac{d \theta_{3}}{d t} & =\frac{\Gamma}{2 C_{3}^{2}}\left(\frac{1}{C_{2}^{2}}-\frac{1}{C_{1}^{2}}\right)+\frac{1}{C_{1}^{2}}
\end{aligned}
$$

To summarize, for the specific choice of vortex numbers and polarities given by Eq. (27), the radial variables $r_{1}, r_{2}$ and $r_{3}$ are expressed in terms of $C_{1}, C_{2}$ and $C_{3}$ from Eqs. (35) and ultimately in terms of $C_{1}$ alone using the conservation laws as in Eqs. (31); while the time evolution of $C_{1}$ is determined from Eq. (33) by a straightforward integration. Similarly, we may use Eqs. (31) to express the right-hand sides of Eq. (36) in terms of $C_{1}$ and thus the time evolution of the angular variables $\theta_{1}, \theta_{2}$ and $\theta_{3}$ is also reduced to simple quadratures.

Without loss of generality, the initial three-vortex configuration is shown in Fig. 1 . In particular, $b$ is the initial length of the base $(1,2)$ of the triangle and $h$ the corresponding height. The impact parameter $a$ is defined as the distance of vortex 2 from the y-axis and is taken to be negative when vortex 2 is located to the left of the $y$-axis and positive otherwise. Also note that 


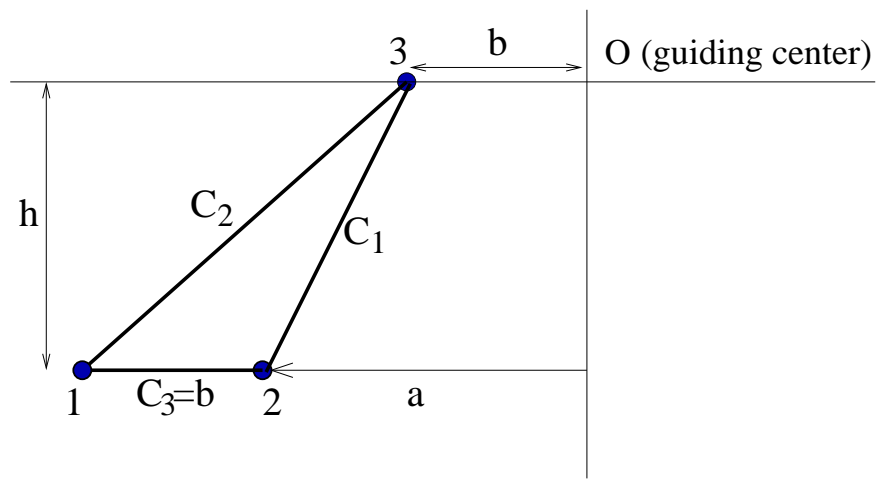

FIG. 1: Initial configuration of three magnetic vortices $(1,2,3)$ with vortex numbers and polarities given by Eq. (27). The origin of the coordinate frame is taken to coincide with the conserved guiding center of the three-vortex system. The impact parameter $a$ is taken to be negative when vortex 2 lies to the left of the y-axis and positive otherwise.

vortex 3 is initially located on the negative $\mathrm{x}$-axis at a distance $b$ from the origin, thus enforcing Eqs. (34). Hence, the initial values of the triangle sides are $C_{1}^{2}=h^{2}+(a+b)^{2}, C_{2}^{2}=h^{2}+a^{2}$, and $C_{3}^{2}=b^{2}$, and the conserved quantities of Eq. (30) are given by

$$
B^{2}=\frac{h^{2}+(a+b)^{2}}{h^{2}+a^{2}} b^{2}, \quad \Gamma=-2 a b,
$$

where $\Gamma$ is positive for left impact $(a<0)$ and negative for right impact $(a>0)$. This completes the description of the general procedure which is explicitly implemented in the continuation of this section using examples of increasing complexity.

\section{A. Special case}

First we employ the general formalism developed in the present paper to recover a special solution already given in the Appendix of our earlier paper [9]. The initial configuration is taken to be an isosceles triangle defined by choosing the impact parameter $a=-b / 2$ in Fig. 1 , The vortex-antivortex pair $(1,2)$ would then move upward with initial velocity $V \approx 1 / b$ (Kelvin motion) and eventually collide against the target vortex 3 . After collision the vortex-antivortex pair moves off to infinity at some scattering angle while the target vortex comes to rest at a new location. Our aim in the following is to calculate the scattering angle as well as the final position of the target vortex.

Now, inserting $a=-b / 2$ in Eq. (37), we find that the values of the conserved quantities are given by

$$
B^{2}=b^{2}=\Gamma
$$

and are independent of the initial triangle height $h$. Then Eqs. (31) read

$$
C_{1}(t)=C_{2}(t), \quad C_{3}(t)=b
$$

which establish that the triangle remains isosceles $\left(C_{1}=C_{2}\right)$ and the length of its base remains constant $\left(C_{3}=b\right)$ at all times $t>0$. But $C_{1}$ itself undergoes a nontrivial time evolution governed 
by Eq. (33):

$$
\frac{d}{d t}\left(C_{1}^{2}\right)=-\nu b\left(\frac{1}{C_{1}^{2}}+\frac{1}{b^{2}}\right) \sqrt{4 C_{1}^{2}-1} .
$$

A more convenient parametrization is obtained by

$$
C_{1}=\sqrt{H^{2}+\frac{1}{4} b^{2}}=C_{2}, \quad C_{3}=b,
$$

where $H=H(t)$ is the instantaneous height of the triangle. Eq. (40) then reads

$$
\frac{d H}{d t}=-\frac{\nu}{b} \frac{H^{2}+\frac{5}{4} b^{2}}{H^{2}+\frac{1}{4} b^{2}}
$$

and must be solved with initial condition $H(t=0)=h$.

During the initial stages of the evolution the three vortices $(1,2,3)$ appear in the plane in a counterclockwise sense and thus $\nu=1$. Eq. (42) is then integrated to yield

$$
\begin{aligned}
& \frac{H}{\zeta b}-\frac{4}{5} \arctan \left(\frac{H}{\zeta b}\right)=\frac{t_{0}-t}{\zeta b^{2}}, \quad 0 \leq t \leq t_{0} \\
& \zeta \equiv \frac{\sqrt{5}}{2}, \quad t_{0} \equiv \zeta b^{2}\left[\frac{h}{\zeta b}--\frac{4}{5} \arctan \left(\frac{h}{\zeta b}\right)\right] .
\end{aligned}
$$

As $t$ approaches $t_{0}$, height $H$ approaches zero and the triangle reduces to a straight line element $(1,3,2)$ at $t=t_{0}$ with vortex 3 located at the center of the line element $(1,2)$. Also recalling that the length of the line element $(1,2)$ remains constant (equal to $b$ ) we conclude that $t_{0}$ is the instance of the closest encounter of the three vortices. For $t>t_{0}$, vortex 3 emerges from the other side and thus Eq. (42) must be integrated with $\nu=-1$ and initial condition $H\left(t=t_{0}\right)=0$ :

$$
\frac{H}{\zeta b}-\frac{4}{5} \arctan \left(\frac{H}{\zeta b}\right)=\frac{t-t_{0}}{\zeta b^{2}}, \quad t>t_{0} .
$$

In the far future $(t \rightarrow \infty) H \rightarrow t / b$ which is consistent with a vortex-antivortex pair $(1,2)$ moving away from the target vortex 3 with asymptotic speed $V=1 / b$, in agreement with Eq. (12)

The preceding results already suggest a process in which the vortex-antivortex pair $(1,2)$ initially approaches the target vortex 3 but eventually moves off to infinity at a scattering angle that remains to be calculated. One must also ascertain the fate of the target vortex 3 well after collision. Both of these questions are answered below by applying Eqs. (35) and (36) to the special case treated in this subsection.

First, we note that Eqs. (35) suggest that the three vortices $(1,2,3)$ together with the origin of the coordinate system $O$ (i.e., the guiding center of the three-vortex system) form a parallelogram $12 O 3$ at all times during the collision. In particular, the third equation (35) establishes that $r_{3}=C_{3}=b$ is time independent, or, vortex 3 moves on a circle whose center coincides with the guiding center and its radius is a constant $b$ equal to the constant length of the base of the triangle. Furthermore, the polar angle $\theta_{3}$ changes during collision by the same amount as the base of the triangle $(1,2)$ rotates in the $2 \mathrm{D}$ plane. Therefore, the total change $\Delta \theta_{3}$ is equal to the scattering angle of the vortex-antivortex pair $(1,2)$ and also specifies the final location of the target vortex 3 .

In order to actually calculate $\Delta \theta_{3}$ in the present special case, we apply Eq. (36) with $C_{1}=C_{2}$ :

$$
\frac{d \theta_{3}}{d t}=\frac{1}{C_{1}^{2}}=\frac{1}{H^{2}+\frac{1}{4} b^{2}},
$$




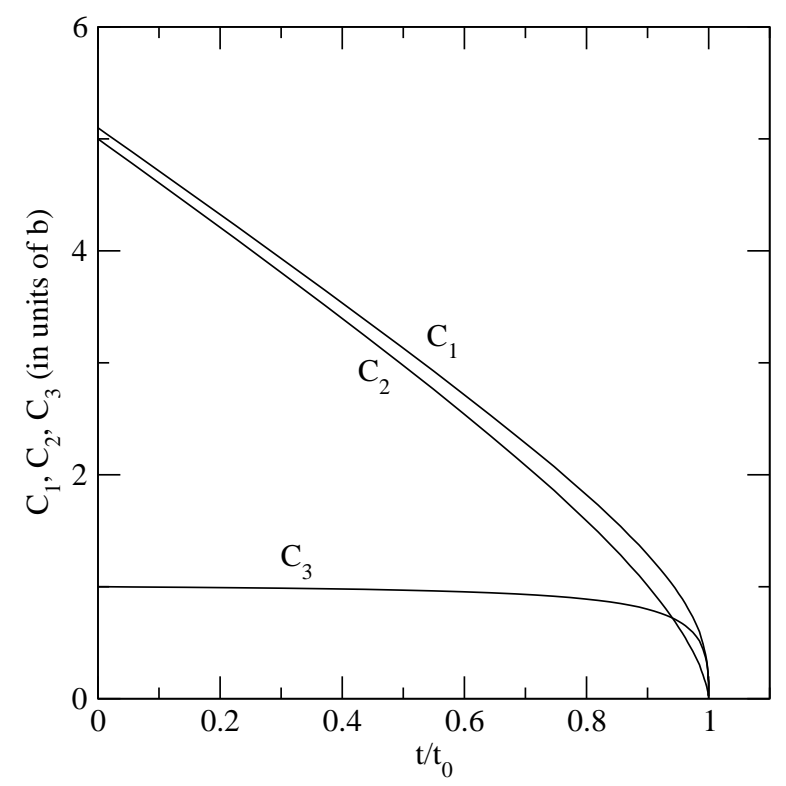

FIG. 2: Time evolution of $C_{1}, C_{2}$ and $C_{3}$ for an initial three-vortex triangle given by Fig. 1 with $a=$ $0, b=1, h=5$. Note that all three lengths simultaneously collapse to zero at a finite time $t_{0}$ calculated from Eq. (53).

where we may insert $H=H(t)$ from Eqs. (43) and (44) to obtain $\theta_{3}=\theta_{3}(t)$ by a further integration. In fact, a more direct calculation of the total scattering angle $\Delta \theta_{3}$ is obtained by combining Eqs. (42) and (45) to write

$$
\frac{d \theta_{3}}{d H}=-\frac{b}{\nu} \frac{1}{H^{2}+\frac{5}{4} b^{2}}
$$

and

$$
\Delta \theta_{3}=b \int_{0}^{h} \frac{d H}{H^{2}+\frac{5}{4} b^{2}}+b \int_{0}^{\infty} \frac{d H}{H^{2}+\frac{5}{4} b^{2}},
$$

where the two terms may be interpreted as the scattering angles before and after collision and correspond to the two branches of the solution $H=H(t)$ given by Eqs. (43) and (44). In particular, for pure scattering where the vortex-antivortex pair originates very far from the target vortex $(h \rightarrow \infty)$ :

$$
\Delta \theta_{3}=2 b \int_{0}^{\infty} \frac{d H}{H^{2}+\frac{5}{4} b^{2}}=\frac{2 \pi}{\sqrt{5}}
$$

which reproduces the special result given in the Appendix of Ref. 9]. A detailed illustration of the actual vortex trajectories may be found in Fig. A1 of the above reference (using a different convention for the origin of the coordinate system) and in Fig. 5 of the present paper (where the origin of the coordinate system coincides with the guiding center). 


\section{B. Three-vortex collapse}

At first sight, the special example treated in the preceding subsection would seem to correspond to a head-on collision of a vortex-antivortex pair off a target vortex. However, such an interpretation seems to be unjustified in view of the peculiar scattering angle calculated in Eq. (48). Indeed, an example that is closer to head-on collision is obtained by choosing vanishing impact parameter $(a=0)$ in the initial vortex configuration shown in Fig. 1]

The initial vortex triangle is then orthogonal, with initial values of the length of its sides $C_{1}(t=0)=\sqrt{h^{2}+b^{2}}, C_{2}(t=0)=h$ and $C_{3}(t=0)=b$. The two conserved quantities of Eq. (30) now read

$$
B=\frac{C_{1} C_{3}}{C_{2}}=\frac{b}{h} \sqrt{h^{2}+b^{2}}, \quad \Gamma=-C_{1}^{2}+C_{2}^{2}+C_{3}^{2}=0 .
$$

A notable consequence of the conservation of $\Gamma=0$ is that the vortex triangle remains orthogonal at all times, even though the length of its sides $C_{1}, C_{2}$, and $C_{3}$, as well as its relative orientation in the $2 \mathrm{D}$ plane, undergo a nontrivial time evolution.

In particular, the evolution of $C_{1}$ is governed by Eq. (33) applied with $\Gamma=0$ and $B$ given by Eq. (49):

$$
\frac{d C_{1}}{d t}=-\frac{C_{1}^{2}+B^{2}}{B C_{1}^{2}}
$$

while $C_{2}$, and $C_{3}$ obtained from Eq. (31) are now given by

$$
C_{2}=\frac{C_{1}^{2}}{\sqrt{C_{1}^{2}+B^{2}}}, \quad C_{3}=\frac{B C_{1}}{\sqrt{C_{1}^{2}+B^{2}}} .
$$

Eq. (50) may easily be integrated to yield

$$
\frac{C_{1}}{B}-\arctan \left(\frac{C_{1}}{B}\right)=\frac{t_{0}-t}{B^{2}}
$$

where the integration constant $t_{0}$ is calculated from the initial condition $C_{1}(t=0)=\sqrt{h^{2}+b^{2}}$, or

$$
t_{0}=\frac{b^{2}}{h^{2}}\left(h^{2}+b^{2}\right)\left[\frac{h}{b}-\arctan \left(\frac{h}{b}\right)\right] .
$$

The physical significance of $t_{0}$ becomes apparent by applying Eq. (52) in the limit $t \rightarrow t_{0}$ :

$$
C_{1}\left(t \rightarrow t_{0}\right) \sim\left[3 B\left(t_{0}-t\right)\right]^{1 / 3}
$$

and Eqs. (51) in the same limit to yield

$$
C_{2}\left(t \rightarrow t_{0}\right) \sim \frac{1}{B}\left[3 B\left(t_{0}-t\right)\right]^{2 / 3}, \quad C_{3}\left(t \rightarrow t_{0}\right) \sim\left[3 B\left(t_{0}-t\right)\right]^{1 / 3} .
$$

Therefore, $t_{0}$ calculated explicitly from Eq. (53) is the instance at which all three $C_{1}, C_{2}$ and $C_{3}$ vanish simultaneously, or, the vortex triangle collapses to a point. Also taking into account Eq. (35), we conclude that the vortex triangle collapses to the guiding center $\left(r_{1}=r_{2}=r_{3}=0\right)$ of the three-vortex system in a finite time interval $t_{0}$. The exact time dependence of $C_{1}, C_{2}$ and $C_{3}$ calculated from Eqs. (52) and (51) over the entire time interval $0 \leq t \leq t_{0}$ is depicted in Fig. 2. 


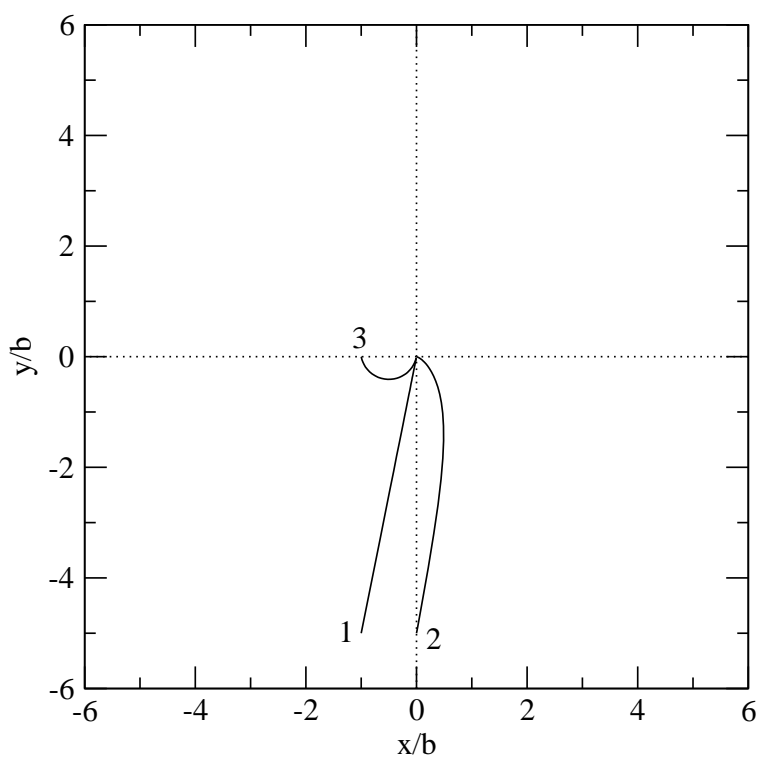

FIG. 3: Trajectories of the three vortices 1,2 and 3 emanating form an initial three-vortex configuration given by Fig. 1 with $a=0, b=1, h=5$. Note that the three trajectories merge at the origin of the coordinate frame which is taken to coincide with the guiding center of the three-vortex system.

Complete information about the vortex trajectories may be obtained by further utilizing the results of Eqs. (35) and (36) or by a direct numerical integration of Eqs. (15). The actual trajectories are illustrated in Fig. 3 which explicitly demonstrates the predicted three-vortex collapse. A notable feature of Fig. 3 is that vortex 1 moves on a straight line that connects its original position with the guiding center of the three-vortex system.

Although this special case of vanishing impact parameter $(a=0)$ is clearly singular from the point of view of scattering theory, it is still possible to calculate the scattering angle $\Delta \theta_{3}$ before collapse. First, we apply the third of Eqs. (36) with $\Gamma=0$ :

$$
\frac{d \theta_{3}}{d t}=\frac{1}{C_{1}^{2}}
$$

and combine this result with Eq. (50) to obtain

$$
d \theta_{3}=-\frac{B d C_{1}}{B^{2}+C_{1}^{2}} .
$$

The scattering angle before collapse is then obtained by integrating Eq. (57) between the values $C_{1}(t=0)=\sqrt{h^{2}+b^{2}}$ and $C_{1}(t=0)=0$ :

$$
\left.\Delta \theta_{3}\right|_{\text {before }}=\int_{0}^{\sqrt{h^{2}+b^{2}}} \frac{B d C_{1}}{B^{2}+C_{1}^{2}}=\arctan \left(\frac{h}{b}\right) .
$$

In the limit where the vortex-antivortex pair originates at a large distance $h \rightarrow \infty$ from the target vortex, we find $\left.\Delta \theta_{3}\right|_{\text {before }}=\pi / 2$ and, by convention, $\left.\Delta \theta_{3}\right|_{\text {total }}=2(\pi / 2)=\pi$. This result agrees with the interpretation of this special case as a head-on collision whose singular nature will also become apparent in the discussion of the following subsection. 


\section{Scattering in the general case}

Our aim in this subsection is to determine the scattering angle as a function of impact parameter $a$. The general strategy is suggested by the special examples treated in the preceding two subsections. Thus we return to the initial configuration depicted in Fig. 1 now applied for arbitraty $a$, which yields values for the conserved quantities $B$ and $\Gamma$ as given in Eq. (377). In particular, we shall be interested in the limit $h \rightarrow \infty$ :

$$
B^{2}=b^{2}, \quad \Gamma=-2 a b,
$$

which corresponds to pure (asymptotic) scattering where the vortex-antivortex pair is initially located very far from the target vortex and also diverges to infinity after collision.

In order to calculate the scattering angle we return to Eq. (36) and express its right-hand side entirely in terms of $C_{1}$ using Eq. (31) to eliminate $C_{2}$ and $C_{3}$. We then combine the resulting equation with Eq. (33) to write

$$
\frac{d \theta_{3}}{d C_{1}^{2}}=-\frac{1}{\nu} \frac{\frac{\Gamma}{2} \frac{B^{2}-\Gamma}{C_{1}^{2}+\Gamma}+\frac{C_{1}^{2}+\Gamma}{C_{1}^{2}+B^{2}} B^{2}}{\sqrt{4 B^{2} C_{1}^{2}\left(C_{1}^{2}+\Gamma\right)^{2}-\Gamma^{2}\left(C_{1}^{2}+B^{2}\right)^{2}}} .
$$

Simple inspection of this equation suggests using scaled variables $u$ and $\gamma$ from

$$
u \equiv \frac{C_{1}^{2}}{B^{2}}, \quad \gamma \equiv \frac{\Gamma}{B^{2}}=-\frac{2 a}{b}
$$

to obtain

$$
\frac{d \theta_{3}}{d u}=-\frac{1}{\nu} \frac{\frac{\gamma(1-\gamma)}{2(u+\gamma)}+\frac{u+\gamma}{u+1}}{\sqrt{4 u(u+\gamma)^{2}-\gamma^{2}(u+1)^{2}}} .
$$

Based on this equation the total scattering angle is calculated as a function of the parameter $\gamma$ alone by a procedure similar to that employed in the special example treated in subsection A:

$$
\Delta \theta_{3}=2 \int_{u_{0}}^{\infty} \frac{\frac{\gamma(1-\gamma)}{2(u+\gamma)}+\frac{u+\gamma}{u+1}}{\sqrt{4 u(u+\gamma)^{2}-\gamma^{2}(u+1)^{2}}} d u
$$

where the factor of two and the upper limit of integration are dictated by the fact that the vortexantivortex pair is located very far from the target vortex well before and after the collision. The lower limit $u_{0}$ is a specific value of $u=C_{1}^{2} / B^{2}$ for which the area of the vortex triangle vanishes. Therefore, $u_{0}$ must be chosen among the three roots of the cubic equation

$$
4 u(u+\gamma)^{2}-\gamma^{2}(u+1)^{2}=0
$$

It is thus important to examine the behavior of the roots as functions of the parameter $\gamma$.

For $\gamma<0$ there exists a real and positive root $u_{1}$ and two complex roots $u_{2}$ and $u_{3}$ such that $u_{2}=u_{3}^{*}$. In this region the lower limit in the integral of Eq. (63) must be chosen as $u_{0}=u_{1}$. Now, $\gamma=0$ is an exceptional point in that all three roots vanish $\left(u_{1}=u_{2}=u_{3}=0\right)$ and thus the lower limit must be chosen as $u_{0}=0$. Applying Eq. (63) for $\gamma=0$ and $u_{0}=0$ yields

$$
\Delta \theta_{3}=\int_{0}^{\infty} \frac{d u}{(u+1) \sqrt{u}}=\pi
$$

in agreement with the conclusion of Subsection B. Indeed, $\gamma=0$ corresponds to vanishing impact parameter $(a=0)$ which is the special case discussed in Subsection B (three-vortex collapse). 
Next we consider the region $0<\gamma<1$ where the cubic equation again possesses a real and positive root $u_{1}$ and two complex roots $u_{2}=u_{3}^{*}$. Thus the lower limit must again be chosen as $u_{0}=u_{1}$. Now, $\gamma=1$ is another exceptional point in that all three roots become real $\left(u_{1}=\frac{1}{4}, u_{2}=-1=u_{3}\right)$ and the lower limit must be chosen to coincide with the positive root $u_{0}=u_{1}=\frac{1}{4}$. Applying Eq. (63) for $\gamma=1$ and $u_{0}=\frac{1}{4}$ yields

$$
\Delta \theta_{3}=2 \int_{\frac{1}{4}}^{\infty} \frac{d u}{(u+1) \sqrt{4 u-1}}=\frac{2 \pi}{\sqrt{5}},
$$

which coincides with the result of Eq. (48). This is not surprising because $\gamma=-2 a / b=1$ leads to an impact parameter $a=-b / 2$ which is indeed the special case discussed in Subsection A.

For $\gamma>1$, the cubic equation again possesses a real and positive root $u_{1}$ and two complex roots $u_{2}=u_{3}^{*}$, thus we must choose $u_{0}=u_{1}$. This generic picture continues to hold until $\gamma$ reaches the critical value

$$
\gamma_{c}=\frac{27}{2}
$$

where all three roots become real $\left(u_{1}=0.5625, u_{2}=9=u_{3}\right)$ and remain real and positive for $\gamma>\gamma_{c}$. In this range of $\gamma$, the three roots may be ordered as $u_{1}<u_{2}<u_{3}$ and the lower limit of integration in Eq. (63) must now be chosen as $u_{0}=u_{3}$.

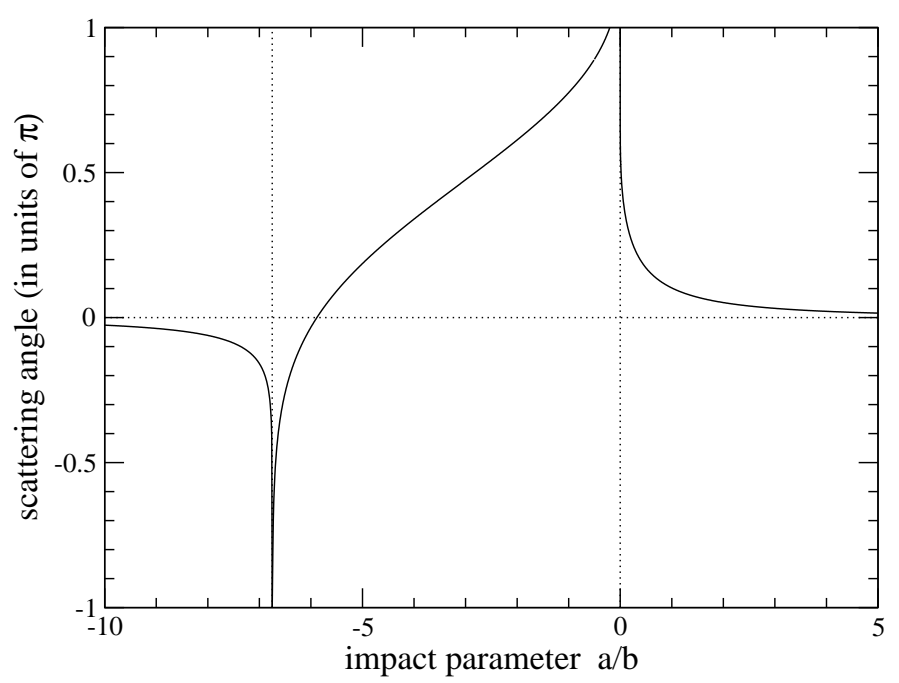

FIG. 4: Scattering angle during collision of a vortex-antivortex (Kelvin) pair $(1,2)$ with a target vortex 3 , as a function of impact parameter $a$. Note the crossover behavior at the critical values $a / b=0$ and $a / b=-27 / 4$ discussed in the text. The results depicted in this figure should be read modulo $2 \pi$.

In all cases the denominator in Eq. (63) does not possess singularities in the domain of integration $\left[u_{0}, \infty\right)$ except for an integrable square root singularity at the lower end. It can also be shown that the numerator in Eq. (63) does not exhibit singularities in the integration domain. Thus we have provided a complete prescription for the calculation of the scattering angle, which entails locating the appropriate root $u_{0}=u_{0}(\gamma)$ of the cubic equation and an elementary numerical integration of the integral in Eq. (63) for any value of $\gamma=-2 a / b$. The calculated scattering angle as a function of impact parameter $a$ measured in units of $b$ is depicted in Fig. 4 .

Thus the scattering angle displays critical (crossover) behavior at two characteristic values of the impact parameter; namely, $a / b=0$, which corresponds to the three-vortex collapse discussed 
in subsection $\mathrm{B}$, and $a / b=-27 / 4$, which corresponds to the critical coupling $\gamma=-2 a / b=27 / 2$ of Eq. (67).
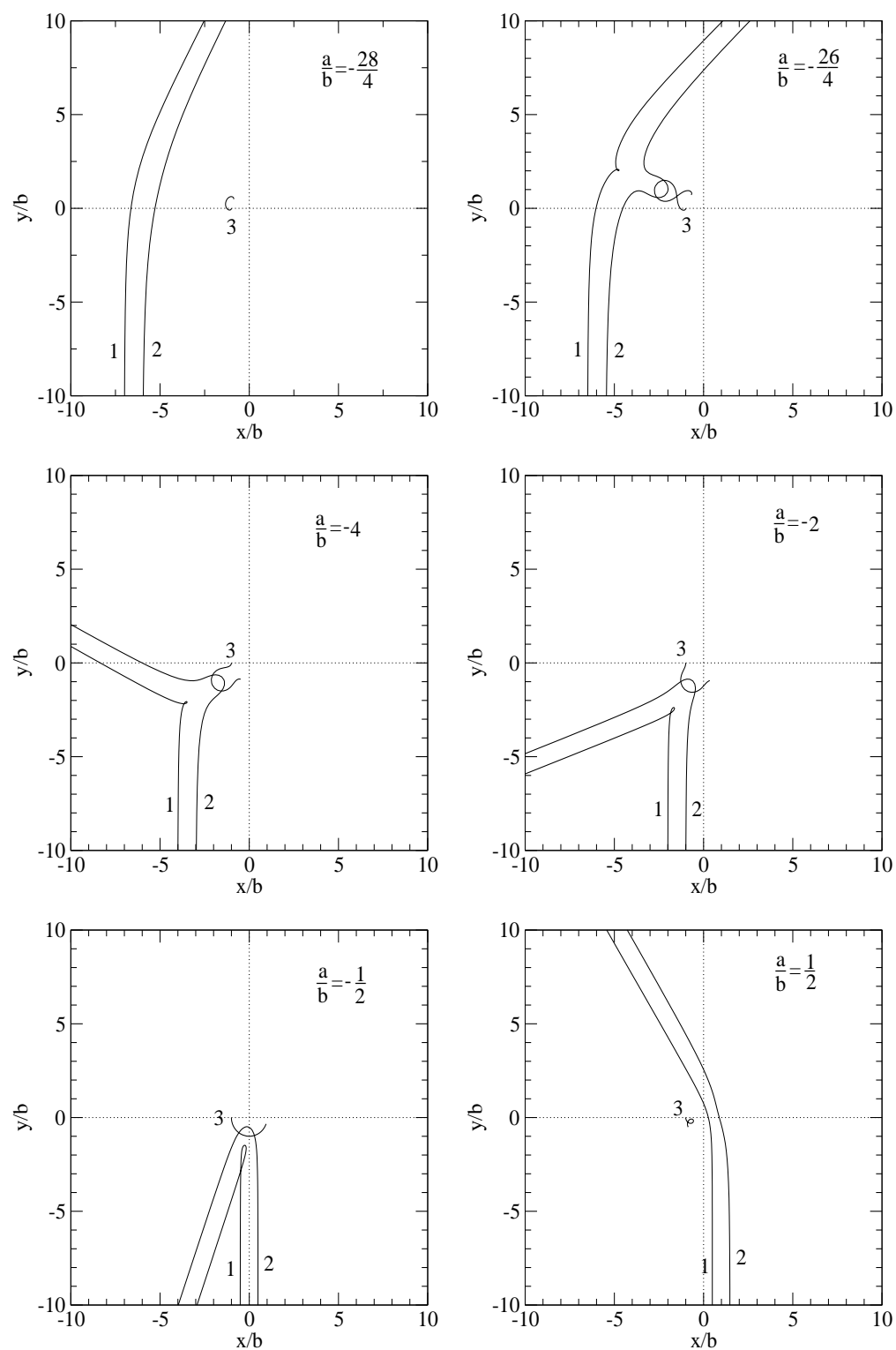

FIG. 5: Vortex trajectories for six characteristic values of the impact parameter $a$. In all cases a vortexantivortex (Kelvin) pair $(1,2)$ collides with a target vortex 3 and is deflected by a scattering angle in agreement with the results of Fig. 4. The target vortex 3 is at rest well before collision and also comes to rest well after collision, as expected for an isolated vortex which is always spontaneously pinned.

In Fig. 5 we display the actual vortex trajectories calculated numerically from Eqs. (15) for a set of six characteristic values of $a / b$. The change of pattern becomes clear as one crosses the two critical values $a / b=0$ and $a / b=-27 / 4$. The nature of the critical parameter $\gamma_{c}$ of Eq. (67) is further illuminated in the following subsection. 


\section{Bounded three-vortex motion}

The analysis of the cubic equation (64) revealed the importance of the critical parameter $\gamma_{c}=27 / 2$ of Eq. (67) above which all three roots are real (and positive). We may then order the roots as $u_{1}<u_{2}<u_{3}$ and note that the argument under the square root in, say, Eq. (62) is positive when either $u>u_{3}$ or $u_{1}<u<u_{2}$. The former case,

$$
\gamma>\gamma_{c}=\frac{27}{2}, \quad u>u_{3}
$$

corresponds to the scattering solution discussed in subsection $\mathrm{C}$ where $u=C_{1}^{2} / B^{2}$ approaches infinity well before or after the collision, while it reaches its minimum value $u_{3}$ at some instance during collision. In contrast, the case

$$
\gamma>\gamma_{c}=\frac{27}{2}, \quad u_{1}<u<u_{2}
$$

may lead to a motion where $u$ oscillates between the values $u_{1}$ and $u_{2}$. Also taking into account Eq. (31) we conclude that all three $C_{1}, C_{2}$ and $C_{3}$ would then remain bounded. The aim of this subsection is to explicitly construct a special class of solutions that realize Eqs. (69) and thus lead to bounded (quasiperiodic) three-vortex motion. In order to achieve the two conditions displayed in Eqs. (69) we consider an initial configuration where the three vortices $(1,2,3)$ lie on a straight line in the order indicated and thus the relative distances are such that $C_{1}+C_{3}=C_{2}$. Specifically, we choose

$$
C_{1}=(2+\epsilon) b, \quad C_{2}=(3+\epsilon) b, \quad C_{3}=b
$$

and initially place the three vortices along the negative $\mathrm{x}$-axis at points such that

$$
\begin{aligned}
& x_{1}=-C_{2}-C_{3}, \quad x_{2}=-C_{2}, \quad x_{3}=-C_{3}, \\
& y_{1}=y_{2}=y_{3}=0 .
\end{aligned}
$$

Then the origin of the coordinate system coincides with the guiding center defined by Eqs. (28). Our aim is to calculate the vortex trajectories emanating from the initial configuration defined by Eqs. (70) and (71).

We first consider the two conserved quantities calculated from such an initial configuration,

$$
B^{2}=\frac{C_{1}^{2} C_{3}^{2}}{C_{2}^{2}}=\left(\frac{2+\epsilon}{3+\epsilon}\right)^{2} b^{2}, \quad \Gamma=-C_{1}^{2}+C_{2}^{2}+C_{3}^{2}=2(3+\epsilon) b^{2},
$$

and thus

$$
\gamma=\frac{\Gamma}{B^{2}}=\frac{2(3+\epsilon)^{3}}{(2+\epsilon)^{2}}
$$

is now the important dimensionless quantity that controls the behavior of the roots of the cubic equation. Note that $\epsilon=0$ leads to the critical parameter $\gamma_{c}=27 / 2$ of Eq. (67) while, interestingly, $\gamma>\gamma_{c}$ for both positive and negative values of the parameter $\epsilon$.

A numerical solution of the initial value problem reveals an interesting picture. For $\epsilon>0$, we encouter a situation of the type described by Eq. (68) which corresponds to a scattering solution where the vortex-antivortex pair $(1,2)$ moves off to infinity leaving behind vortex 3 which relocates to a new final position. However, $\epsilon<0$ leads to a situation of the type anticipated by Eq. (69). The resulting bounded (quasiperiodic) three-vortex motion is illustrated in Fig. 6 for $\epsilon=-1 / 4$. 


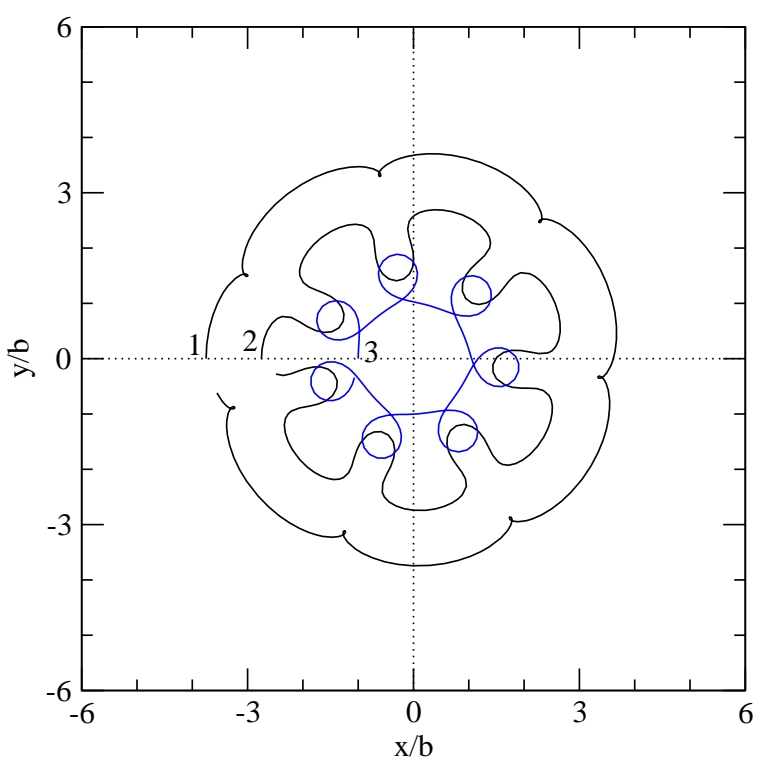

FIG. 6: Trajectories of three vortices in bounded (quasiperiodic) motion emanating from an initial configuration specified by Eqs. (70) and (71) with $\epsilon=-\frac{1}{4}$.

Although the present calculation is performed in an infinite magnetic film, this type of bounded motion may be relevant for the dynamics in finite, disc-shaped ferromagnetic elements.

In the special limit $\epsilon \rightarrow 0$ the three vortices lie on a straight line for all times at constant relative distances:

$$
C_{1}=2 b, \quad C_{2}=3 b, \quad C_{3}=b .
$$

Such a linear configuration rotates rigidly around a fixed guiding center with constant angular frequency

$$
\omega=\frac{d \theta_{1}}{d t}=\frac{d \theta_{2}}{d t}=\frac{d \theta_{3}}{d t}=-\frac{1}{6 b^{2}}
$$

a relation obtained by applying Eqs. (36) to the present degenerate case.

\section{CONCLUSIONS}

We have studied the equations of motion for magnetic vortices which are approximated as point vortices. These are a generalization of the Helmholtz-Kirchhoff equations [10, 11] derived for ordinary fluid vortices. They differ from them in that magnetic vortices have an additional characteristic, their polarity, which enters in the equations. The main body of the paper is devoted to the case of three magnetic vortices which constitute an integrable system. For the integration of this system we follow the steps of the seminal paper of Gröbli [12] modified to include the vortex polarity.

Magnetic vortices are commonly observed in magnetic elements with typical dimensions of the order of a few micrometers or hundreds of nanometers. A single vortex spontaneously created at the center of an element can be driven by a magnetic field or an electrical current to produce excitations in its vicinity. It has been observed in various numerical simulations that such 
excitations often have the form of a lump of magnetization opposite to that of the original vortex. In some cases the lumps are amplified to become vortex-antivortex pairs with clearly distinct constituent vortices. Such a process was apparently observed in experiments where the original vortex was driven by a magnetic field [3, 4] or an electrical current [5]. The fact that the created vortex-antivortex pair has opposite polarity to the original vortex should most probably be attributed to the effect of the magnetostatic (dipole-dipole) interaction which is the only magnetic interaction that primarily favors domains of opposite magnetization. From these examples it follows that the three-vortex configuration, given in Eq. (27), on which we have mainly focused in this paper, is a particularly interesting one for the purposes of understanding vortex dynamics in magnetic elements.

In view of the above comments it is interesting to compare our results with simulations of the Landau-Lifshitz equation. We have used the Landau-Lifshitz equation with an exchange interaction and an on-site easy-plane anisotropy so that vortices are the relevant excitations of the system. Some results have been published in Refs. [8, 9]. For the case of vortex-antivortex pairs where the vortices are well-separated the simulations show very good agreement with the analytical solutions for the vortex trajectories. It is worth discussing separately the special case of three-vortex collapse of Subsection IVB which would appear to be specific to the point vortex system. We have simulated the case of zero angular momentum within the LandauLifshitz equation and have observed vortex trajectories very similar to those shown in Fig. 3, The original vortex and the antivortex are annihilated when the three-vortex system collapses to the origin and an isolated vortex 1 is the final outcome of the simulation. The results of the simulation are closer to the analytical solutions when the vortex-antivortex pair is large, i.e., the vortex and the antivortex are initially well separated.

\section{Aknowledgements}

We are grateful to Mahir Hussein for stimulating discussions that refocused our interest on this subject.

[1] A. P. Malozemoff and J. C. Slonczewski, "Magnetic Domain Walls in Bubble Materials" (Academic Press, New York, 1979).

[2] D. L. Huber, Phys. Rev. B 26, 3758 (1982).

[3] A. Neudert, J. McCord, R. Schäfer, and L. Schultz, J. Appl. Phys. 97, 10E701 (2005).

[4] B. V. Waeyenberge, A. Puzic, H. Stoll, K. W. Chou, T. Tyliszczak, R. Hertel, M. Fähnle, H. Brückl, K. Rott, G. Reiss, I. Neudecker, D. Weiss, C. H. Back, and G. Schütz, Nature(London) 444, 461 (2006).

[5] K. Yamada, S. Kasai, Y. Nakatani, K. Kobayashi, H. Kohno, A. Thiaville, and T. Ono, Nature Materials 6, 269 (2007).

[6] N. Papanicolaou and T. N. Tomaras, Nucl. Phys. B 360, 425 (1991).

[7] S. Komineas and N. Papanicolaou, Physica D 99, 81 (1996).

[8] S. Komineas and N. Papanicolaou, "Dynamics of vortex-antivortex pairs in ferromagnets" in E. Kamenetskii (ed.), "Electromagnetic, Magnetostatic, and exchange-interaction vortices in confined magnetic structures", (Transworld Research Networks, 2008); arXiv:0712.3684v1

[9] S. Komineas and N. Papanicolaou, New J. Phys. 10, 043021 (2008).

[10] H. Helmholtz, J. Reine Angew. Math. 55, 22 (1858) [P. G. Tait, Philos. Mag. 33, 485-510 (1867)].

[11] Kirchhoff, "Vorlesungen über mathematische Physik. Mechanik" (Teubner, Leipzig, 1876).

[12] W. Gröbli, "Spezielle Probleme ber die Bewegung geradliniger paralleler Wirbelfäden" (Zürcher and Furrer, Zürich, 1877). Reprinted in Vierteljahrsschr. Natforsch. Ges. Zur. 22, 37-81 (1877); ibid. 22, 129-165 (1877). 
[13] H. Aref, N. Rott, and H. Thomann, Annu. Rev. Fluid Mech. 24, 1 (1992).

[14] H. Aref, Phys. Fluids 22, 393 (1979).

[15] A. A. Thiele, Phys. Rev. Lett. 30, 230 (1973).

[16] V. L. Pokrovskii and G. V. Uimin, JETP Lett. 41, 128 (1985).

[17] A. Kovalev, S. Komineas, and F. G. Mertens, Eur. Phys. J. B 65, 89 (2002). q

[18] N. Papanicolaou and P. N. Spathis, Nonlinearity 12, 285 (1999).

[19] S. Komineas, Phys. Rev. Lett. 99, 117202 (2007). 